\title{
Post-reproductive Health: Window of Opportunity for Preventing Comorbidities
}

\author{
Suvarna Satish Khadilkar ${ }^{1}$
}

Received: 11 January 2019/Accepted: 12 January 2019/Published online: 25 January 2019

(C) Federation of Obstetric \& Gynecological Societies of India 2019

\begin{abstract}
About the Author

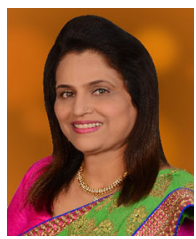

Prof. Suvarna Satish Khadilkar is the Editor in Chief of this journal. She is currently working as Consultant GyneEndocrinologist and Professor and Head of the Department of Obstetric and Gynecology, Bombay Hospital Institute of Medical Sciences and Medical Research Centre, Mumbai. In the past, she worked as an Associate Professor and Unit Chief at JJ Group of Hospitals and Grant Medical College [GMC], Mumbai, and thereafter in the capacity of the Professor and Head in the Department of Obstetrics and Gynecology, Government Medical College, Kolhapur, Maharashtra. She is an undergraduate and postgraduate teacher and examiner, Mumbai University and Maharashtra University of Health Sciences over past 30 years. Pursuing her interest in endocrinology, she acquired Diploma in Endocrinology from the prestigious University of South Wales, UK, and has been appointed as a recognized teacher in endocrinology in the University of South Wales. She has been appointed as a member of FIGO working group on post-reproductive health. She is the Treasurer of FOGSI. She has held many prestigious positions like President of Indian Menopause Society, Chairperson of Reproductive Endocrinology Committee of FOGSI, President, Association of Medical Women in India, Mumbai. She is currently the librarian of Mumbai Ob-Gyn Society. She has published six textbooks and more than 70 articles at national and international levels. She is recipient of 30 awards for her research work including the Young Scientist Award.
\end{abstract}

\begin{abstract}
Post-reproductive health is a challenging issue as it is surrounded by many controversies. Menopausal
\end{abstract}

Prof. Suvarna Satish Khadilkar MD DGO FICOG, CIMP, Diploma in Endocrinology (UK) is Editor in Chief of Journal of Obstetrics and Gynecology of India, and Treasurer, FOGSI; she is Consultant GyneEndocrinologist and Professor and Head of the Dept of Ob-Gyn, Bombay Hospital and Medical Research Centre, Mumbai, Former Professor and Head, Dept of Ob-Gyn, RCSM, Government Medical College, Maharashtra, and Associate Professor and Unit Chief Grant Medical College and Cama and Albless Hospital, Mumbai.

Suvarna Satish Khadilkar suvarnakhadilkar@yahoo.com; suvarnakhadilkar2@gmail.com

1 Bombay Hospital and Medical Research Centre, 12 New Marine Line, Mumbai 400020, India hormone therapy (MHT) has witnessed a rise and fall over last several years. We do understand that comorbidities incidence rises beyond menopause and we need to prevent them. When MHT was used universally, the risk-benefit ratio became unfavorable. Hence, the use of MHT dropped drastically. But newer studies have introduced the concept of window of opportunity, wherein MHT does not have harmful effects, but in fact it will prevent comorbidities associated with menopause. This editorial focuses on the prevention of menopause- and MHT-related comorbidities.

Keywords Post-reproductive health - Menopause . HRT $\cdot$ MHT $\cdot$ Window of opportunity 


\section{Burden of the Post-reproductive Health}

Millions of women face health challenges beyond menopause worldwide, and a significant proportion of them live in India. As per 2011 census, total population of India is 1.21 billion. $48.46 \%$ of women contribute to total population of India. Life expectancy at birth for females in India is projected to be 72.3 years, whereas the average age of menopause is only 46 years [1]. Hence, it is important to maintain positive post-reproductive health, prevent diseases, reduce the mortality rates and improve quality of life. While treatment with menopausal hormone therapy (MHT) has documented benefits, it is associated with some risks like breast cancer and thromboembolism which will worsen the already increased risks pertaining to age- and menopause-related morbidity. This editorial will focus on how to prevent comorbidities beyond menopause, and whether intervention with MHT in the window of opportunity will minimize risks associated with MHT.

\section{Endogenous Estrogen and Comorbidities}

Endogenous estrogen has many beneficial and protective effects which delay onset of age-related comorbidities. Due to decline in the levels of endogenous estrogen at menopause, the risk of vasomotor symptoms, osteoporosis, cardiovascular diseases, malignancy and dementia increases. Estrogen prevents atherosclerosis and inflammatory changes and is more lipid friendly (HDL and LDL), thus preventing cardiovascular events. Estrogen acts on vascular endothelium and smooth muscles which causes vasodilatation, preventing cardiovascular risks.

\section{Comorbidities Associated with Menopause}

Worldwide, cardiovascular diseases are the leading causes of morbidity and mortality. It has been observed that cardiovascular diseases risk increases with age in men as well as in women. However in women due to cardio-protective effect of endogenous estrogen, the risk is delayed till menopause. Cancer- and osteoporosis-related fractures are two other major morbidities. Cerebrovascular events, pulmonary embolism, dementia, breast cancer and endometrial cancer may occur as a side effect of MHT.

An account of various comorbidities is given in Table 1.

Since incidence of heart disease in women is higher after menopause, exogenous hormones containing estrogens/progesterone were used, with the aim of reducing the risk of heart disease. Menopausal women were prescribed MHT universally before results of Women's Health
Table 1 Morbidities related to age, menopause and MHT

Cardiovascular morbidities
Cerebrovascular morbidities
Deep venous and pulmonary embolism
Dyslipidemia
Diabetes
Dementia
Breast cancer
Endometrial cancer
Ovarian cancer
Cervical cancer
Osteoporosis-related fractures

Initiative (WHI) [2] were published. The Women's Health Initiative (WHI) trial and Heart and Estrogen Progestin Replacement (HERS) [3] study both concluded that MHT is not recommended for primary or secondary prevention of coronary heart disease. The universal use of MHT following menopause fells drastically thereafter.

Even though the WHI trial was stopped after 7 years, 27,347 postmenopausal women aged between 50 and 79 years who were included in the trial were followed up for an extended period of 13 years with no treatment. Further analysis of the data gave many important insights.

With the awareness of issues related to MHT, currently postmenopausal women seek help only if they are having symptoms, but some may seek help to keep them young forever and improve the quality of life. The universal use of MHT is not recommended anymore, so how do we select women for prescribing MHT to control symptoms, prevent comorbidities and give them better quality of life? What strategy should we use to achieve the best balance between risks and benefits of MHT? Answers to these questions are not very clear, but recently introduced concept of window of opportunity may be useful in achieving goals.

\section{Cardiovascular Diseases: Window of Opportunity}

A concept of "timing hypothesis" or "window of opportunity" has been introduced recently for the initiation of menopausal hormone therapy. It indicates that MHT is best given immediately postmenopause for a short duration of time. MHT started many years after menopause and continued for long duration, and thereafter, has been associated with more side effects. WHI data analysis showed that MHT was not associated with higher complications among younger age group (50-59) when analyzed separately [4].

The Danish Osteoporosis Prevention Study (DOPS) $[5,6]$ and the Kronos Early Estrogen Study (KEEPS) [7] have generated interest in MHT due to encouraging results. 
The Danish Osteoporosis Prevention Study (DOPS) was initiated in 1990 for a duration of 20 years. It was a partly randomized study and included normal and healthy postmenopausal women. It concluded that MHT has a beneficial effect on coronary artery disease rather than worsening it. The study supported the fact that hormonal therapy initiated immediately after menopause (up to 7 months postmenopause) reduces mortality due to coronary disease significantly. It also demonstrated reduced incidence of heart failure and myocardial infarction. Most importantly, they also proved that the risk of thromboembolic events, stroke or cancer was not increased with MHT.

The Kronos Early Estrogen Study (KEEPS) was a double-blind, randomized, placebo-controlled multi-centric trial. It lasted for 4 years, in which 728 women with an average age of 50 years were enrolled within 3 years of menopause. It assessed the progression of carotid intimamedia thickness and atherosclerosis by using the coronary artery calcium score. They found that the use of MHT did not worsen carotid intima-media thickness or lead to higher incidence of atherosclerosis. The study also supports the concept of a window of opportunity in the early postmenopause when the initiation of MHT has a net beneficial effect. As per global consensus [8] MHT does reduce the risk of the cardiovascular disease only if it is used in this period of "window of opportunity." Hence to reduce coronary heart disease risk and overall mortality in women, the best strategy would be to initiate hormonal therapy within 10 years of menopause and/or before 60 years of age, and continue for a short duration of time. MHT after 60 years has a risk, as with advancing age, the vascular lumen gets partially obliterated with atherosclerotic plaques. MHT will make the plaque unstable, and it reduces the vasodilatation which will eventually increase the risk of cardiovascular diseases. ELITE trial also supports this timing hypothesis [9].

The 2017 Cochrane Collaboration systematic review studied the effects of using MHT for 1 year or more [10]. This review covered twenty-three randomized double-blind studies, involving 43,637 women. Authors concluded that MHT for primary or secondary prevention of CVD or for preservation of cognitive function was not indicated. It should be noted that only about $30 \%$ of women from this review were 50 to 59 years old at baseline, analysis of these data of younger women showed that at the end of 2 years, only venous thromboembolism incidence increased, whereas no other risk was noted.

The conclusion of all the above-mentioned studies indicates that initiating MHT early, before atherosclerosis has established will help delay its further progression.

\section{Are Vasomotor Symptoms Biomarkers of Cardiovascular Diseases?}

The evidence suggests that vasomotor symptoms could be looked upon as biomarkers of cardiovascular diseases [11]. It is well established that MHT including tibolone and bazedoxifene is very effective for controlling vasomotor symptoms (VMS) associated with menopause at any age. Comorbidities can be prevented if MHT is initiated for symptomatic women before the age of 60 years or within 10 years after menopause. Non-hormonal options may be explored only if MHT is contraindicated, or patient is not willing for the same. The current data seem to indicate that vasomotor symptoms could be a biomarker for cardiovascular diseases. Perhaps, this will help the selection of patients for longer duration of MHT.

\section{Vasomotor Symptoms and Osteoporosis: Any Correlation?}

Latest evidence suggests that all women having vasomotor symptoms also tend to have lower bone mineral density. Medical records of more than 23,000 US women aged 50-79 years were studied by a group of investigators. Results showed that "women with moderate/severe VMS had lower bone mineral density at the femoral neck and lumbar spine and increased rates of hip fractures during more than 8 years of follow-up compared with women who did not have VMS" [12]. So we could focus on these symptomatic women for the prevention of osteoporosisrelated morbidity and mortality. It is well accepted and proved in various randomized controlled trials that MHT including tibolone and bazedoxifene lowers the risk of fractures related to osteoporosis in postmenopausal women. MHT can be initiated in postmenopausal women at risk of fracture or osteoporosis in window of opportunity, but initiation after the age of 60 years requires individualized approach.

\section{Mood Changes and MHT}

MHT helps improve mood changes in early postmenopausal women. MHT may also be useful for perimenopausal women with major depression. Some women with suicidal tendencies feel better with MHT, but antidepressant therapy must be the first choice in this setting in consultation with a psychiatrist. 


\section{Reducing MHT-Related Comorbidities}

There is a large amount of high-quality evidence that proves that estrogen-alone MHT decreases incidence of myocardial infarction and also all-cause mortality when initiated in women younger than 60 years of age and/or within 10 years of menopause. But estrogen-alone therapy is not recommended when uterus is intact, so addition of progesterone is mandatory to prevent occurrence of endometrial cancer.

The evidence on estrogen plus progestogen therapy initiated in women younger than 60 years of age or within 10 years of menopause shows varied results compared to the estrogen-alone group. To reduce progesterone-related morbidity, strategy of using lipid-friendly molecules like natural progesterone and dydrogesterone may be preferred. However, the risk of arterial and venous thromboembolism (VTE) has not been reduced with oral MHT.

The increased risk of breast cancer is associated with the use of a medroxyprogesterone. More data on other molecules of progestins will help plan further strategies.

MHT initiated in early menopause has shown some evidence that it may prevent Alzheimer's disease in later life [13]. Oral MHT initiated in older women has demonstrated increased risks of dementia.

\section{Type, Route and Duration of MHT}

Global consensus recommends that MHT should be used in smallest possible dose and shortest possible duration before the age of 60 or within 10 years of menopause. But women who have VMS beyond the age of 60 may require MHT. If needed beyond the age of 60 , then it should be practiced only after doing individual risk calculation to keep the morbidities to minimum. A recent study changed the belief that VMS are short lasting and disappear within 2 years. VMS can last for many years. So, it may not be suitably included in "short-term symptoms" of menopause.

The study showed that VMS lasted in excess of 7 years for more than 50\% women in the SWAN study persisting for 4.5 years after the final menstrual period [14]. A population-based, cross-sectional study of 1,548 older Australian women between 65 and 79 years showed that VMS prevalence was $39.2 \%$ and the highest prevalence of VMS was observed in women aged between 65 and 69 years [15]. The American College of Obstetricians and Gynecologists recommends against routine discontinuation of systemic estrogen at the age of 65 years.

The dose, type and route of MHT should be selected as per treatment goals, patient's choice and risk factors; hence, it should be individualized. The risk profile needs to be individually reassessed annually. Some women may require it for longer duration.

The decision about how and when to start MHT and when to stop MHT must be made after discussing in detail with the patient and must be carried out under expert supervision. Hormonal therapy may be harmful in women having preexisting coronary disease, cerebrovascular disease or thromboembolic tendencies. In the presence of high-risk factors if MHT is indicated, transdermal preparations are preferred. Women need to be aware of the nonhormonal therapies available for both management of vasomotor symptoms associated with perimenopause and early menopause. Premature menopause requires standard dose of MHT prescribed till the age of natural menopause and discontinued thereafter with an informed decision.

\section{Conclusion}

In the current scenario, MHT should not be used routinely for the primary or secondary prevention of CHD. The opportunity window or timing hypothesis has cardiovascular benefits when MHT is initiated immediately after menopause. The role of natural progesterone versus synthetic progestins needs further evaluation. Healthy lifestyles and alternative strategies should be encouraged to reduce cardiovascular risk in menopausal women. Prescription of MHT does improvise quality of life. The initiation, route and duration of MHT should be individualized for each patient after adequate counseling and thorough discussion of risk-benefit ratio. An early start of MHT in selected women in window of opportunity is the key to have best balance of risks and benefits for preventing all comorbidities related to menopause and MHT.

\section{References}

1. 2011 census statement. http://www.censusindia.gov.in/ 2011census/. Ministry of Home Affairs, Government of India. Accessed 12 Jan 2019.

2. Writing Group for the Women's Health Initiative. Investigators risks and benefits of estrogen plus progestin in healthy postmenopausal women: principal results from the women's health initiative randomized controlled trial. JAMA. 2002;288:321-33.

3. Grady D, Brown JS, Vittinghoff E, et al. HERS Research Group. Postmenopausal hormones and incontinence: the Heart and Estrogen/Progestin Replacement Study. Obstet Gynecol. 2001;97:116-20.

4. Rossouw JE, Prentice RL, Manson JE, et al. Postmenopausal hormone therapy and risk of cardiovascular disease by age and years since menopause. JAMA. 2007;297(13):1465-77.

5. Mosekilde L, Hermann AP, Beck-Nielsen H, et al. The Danish Osteoporosis Prevention Study (DOPS): project design and inclusion of 2000 normal perimenopausal women. Maturitas. 1999;31(3):207-19. 
6. Schierbeck LL, Rejnmark L, Tofteng CL, et al. Effect of hormone replacement therapy on cardiovascular events in recently postmenopausal women: randomised trial. BMJ. 2012;9(345):e6409.

7. Wolff EF, He Y, Black DM, et al. Self-reported menopausal symptoms, coronary artery calcification, and carotid intima-media thickness in recently menopausal women screened for the Kronos early estrogen prevention study (KEEPS). Fertil Steril. 2013;99(5):1385-91.

8. De Villiers TJ, Hall JE, Pinkerton JV, et al. Revised global consensus statement on menopausal hormone therapy. Climacteric. 2016;19:313-5.

9. Hodis HN, Mack WJ, Henderson VW, et al. Vascular effects of early versus late postmenopausal treatment with estradiol. N Engl J Med. 2016;374(13):1221-31.

10. Marjoribanks J, Farquhar C, Roberts H, et al. Long-term hormone therapy for perimenopausal and postmenopausal women. Cochrane Database Syst Rev. 2017. https://doi.org/10. 1002/14651858.cd004143.pub5.

11. Biglia N, Cagnacci A, Gambacciani M, et al. Vasomotor symptoms in menopause: a biomarker of cardiovascular disease risk and other chronic diseases? Climacteric. 2017;20(4):306-12.
12. Crandall CJ, Aragaki A, Cauley JA, et al. Associations of menopausal vasomotor symptoms with fracture incidence. J Clin Endocrinol Metab. 2015;100:524-34.

13. Wharton W, Gleason CE, Miller VM, et al. Rationale and design of the Kronos Early Estrogen Prevention Study (KEEPS) and the KEEPS cognitive and affective sub study (KEEPS Cog). Brain Res. 2013;13(1514):12-7.

14. Avis NE, Crawford SL, Greendale G, et al. Duration of menopausal vasomotor symptoms over the menopause transition. JAMA Intern Med. 2015;175:531-9.

15. Zeleke BM, Bell RJ, Billah B, et al. Vasomotor and sexual symptoms in older Australian women: a cross-sectional study. Fertil Steril. 2016;105:149-55.

\section{Publisher's Note}

Springer Nature remains neutral with regard to jurisdictional claims in published maps and institutional affiliations. 